\title{
Serotonergic Dysfunction in Patients with Bipolar Disorder Assessed by the Loudness Dependence of the Auditory Evoked Potential
}

\author{
Kyung-Sang Lee ${ }^{1,2}$, Young-Min Park ${ }^{1,2}$ and Seung-Hwan Lee ${ }^{1,2} \bowtie$ \\ 'Department of Psychiatry, Inje University College of Medicine, Ilsan Paik Hospital, Goyang, Republic of Korea \\ ${ }^{2}$ Clinical Emotion and Cognition Research Laboratory, Goyang, Republic of Korea
}

\begin{abstract}
Objective The loudness dependence of the auditory evoked potential (LDAEP) is suggested to be a marker of serotonin system function. This study explored the LDAEP of multiple mood statuses (depression, mania, and euthymia) and its clinical implication in bipolar disorder patients.
\end{abstract}

Methods A total of 89 subjects, comprising 35 patients with bipolar disorder, 32 patients with schizophrenia, and 22 healthy controls were evaluated. The bipolar disorder cases comprised 10 depressed patients, 15 patients with mania, and 10 euthymic patients. The N1/ P2 peak-to-peak amplitudes were measured at 5 stimulus intensities, and the LDAEP was calculated as the slope of the linear regression. Both cortical and source LDAEP values were calculated.

Results LDAEP varied according to mood statuses, and was significantly stronger in cases of euthymia, depression, and mania. Cortical LDAEP was significantly stronger in patients with bipolar euthymia compared with schizophrenia, stronger in bipolar depression than in schizophrenia, stronger in healthy controls than in schizophrenia patients, and stronger in healthy controls than in patients with bipolar mania. Source LDAEP was significantly stronger in patients with bipolar euthymia, bipolar depression, and bipolar mania compared with schizophrenia, stronger in bipolar euthymia than in bipolar mania. Psychotic features weakened the source LDAEP relative to nonpsychotic features. The severity of the depressive symptom was negatively correlated with source LDAEP.

Conclusion These findings suggest that the serotonin activity of patients with bipolar disorder may vary according to mood status. A longitudinal follow-up study should be pursued using drug-naive subjects.

Psychiatry Investig 2012;9:298-306

Key Words LDAEP, Bipolar disorder, Mood status, Serotonin.

\section{INTRODUCTION}

The loudness dependence of the auditory evoked potential (LDAEP) has been proposed as a reliable indicator of the central serotonin (5-HT) system in humans. ${ }^{1-3}$ It is measure of activity in the cerebral cortex that is reflected by a change in the amplitude of the N1/P2 component of the auditory evoked potential with increasing auditory tone loudness. ${ }^{1}$ The serotonergic neurons that innervate the auditory cortex, particu-

Received: April 24, 2012 Revised: June 15, 2012

Accepted: June 17, 2012 Available online: September 6, 2012

$\triangle$ Correspondence: Seung-Hwan Lee, MD, PhD

Department of Psychiatry, Inje University College of Medicine, Ilsan Paik Hospital, 2240 Daehwa-dong, Ilsanseo-gu, Goyang 411-706, Republic of Korea

Tel: +82-31-910-7260, Fax: +82-31-919-9776

E-mail: 1shpss@paik.ac.kr, lshpss@hanmail.net

(a) This is an Open Access article distributed under the terms of the Creative Commons Attribution Non-Commercial License (http://creativecommons.org/licenses/by$\mathrm{nc} / 3.0$ ) which permits unrestricted non-commercial use, distribution, and reproduction in any medium, provided the original work is properly cited. larly the primary auditory cortex, are thought to modulate this relation-whereby greater increases in amplitude represent diminished serotonin function, and smaller increases in amplitude represent increased serotonin function. ${ }^{1}$

The affective switch, which may occur upon treating bipolar patients with serotonergic drugs, implies that the serotonin system contributes to the pathology of bipolar disorder. ${ }^{4-6}$ Antidepressants that target the serotonergic system have been associated with varying degrees of propensity to induce a treatment-emergent affective switch, providing valuable evidence that mood-switch processes are related to central serotonergic function. ${ }^{4}$ Lee et al. ${ }^{5}$ have reported 2 patients who exhibited aberrantly high LDAEP, who also exhibited mood elevation and side effects in response to selective serotonin reuptake inhibitor (SSRI) antidepressants. Park et al. ${ }^{6}$ reported a patient with major depressive disorder who exhibited a low baseline LDAEP value and experienced severe adverse effects (e.g., 
nausea and vomiting, generalized weakness, headache, akathisia, and anxiety) after the administration of SSRIs, without improvement of his depressive symptoms. There are several reports that strong LDAEP is associated with a positive treatment response in patients with bipolar disorder who have taken lithium. ${ }^{7-9}$ A strong LDAEP is thus associated with a deficit in serotonergic neurotransmission. ${ }^{10}$

The contribution of the serotonin system to bipolar disorder is supported by genetic evidence. ${ }^{11-14}$ Mundo and colleagues ${ }^{11}$ found that the short-allele polymorphism of the serotonin transporter gene (5-HTTLPR) promoter was overrepresented in patients who developed treatment-emergent hypomania or mania after receiving SSRIs. There is some controversy regarding the levels of the serotonin reuptake transporter (SERT) in the prefrontal cortex of patients with bipolar disorder. ${ }^{12}$ Some studies indicate no change in the density of SERT in the postmortem prefrontal cortex of patients with bipolar disorder. ${ }^{13}$ Using a serial analysis of gene expression, another study reports elevated SERT expression in the frontal cortex of patients with bipolar disorder. ${ }^{14}$

Mahmood and Silverstone ${ }^{15}$ have reviewed evidence that suggests that serotonin is likely to play a pivotal role in the pathophysiology of bipolar disorder. However, patients with bipolar disorder have exhibited mixed findings of serotonin activity or availability according to their mood status. Absolute levels of the 5-HT metabolite 5-hydroxyindoleacetic acid (5HIAA), are reduced in the CSF during bipolar depression ${ }^{16}$ and increased during mania. ${ }^{17}$ Significantly reduced hypothalamopituitary axis response to 5-HT agonists (D-fenfluramine) is found in bipolar mania. ${ }^{18}$ Diminished cortisol response to a tryptophan challenge has been reported in bipolar euthymia. ${ }^{19}$

To our knowledge, few studies have specifically addressed the LDAEP of patients with bipolar disorder. In this study, we compared LDAEP strength among bipolar disorder, schizophrenia, and healthy controls. The current study also addresses the question of whether serotonergic activity differs among bipolar disorder with different mood statuses (depression, mania, and euthymia).

\section{METHODS}

\section{Subjects}

A total of 97 subjects were recruited, of whom 89 participants remained after the exclusion of 8 individuals (bipolar disorder: 5 , healthy controls: 3 ) whose LDAEP measurements were greater than 2 standard deviations outside the entire LDAEP dataset.

A total of 89 subjects between the ages of 18 and 61 years participated in this study. Of these, 35 were patients with bipolar disorder, 32 were patients with schizophrenia, and the remainder ( $n=22)$ were healthy controls. Psychiatric diagnoses were made on the basis of the Structured Clinical Interview for DSM-IV Axis I Disorders (SCID-I) ${ }^{20}$ and Axis II Disorders (SCID-II). ${ }^{21}$ The interviews were conducted face-to-face by psychiatrists and psychiatric residents who had received training in the use of the instrument. Healthy controls were recruited from the local community through newspaper advertisement and fliers. After initial screening, subjects were excluded if they had a family history of psychiatric disorders. Potential healthy controls were interviewed using the SCIDII and were excluded if they have had any of the disorders being studied. Subjects with any severe medical illness, high suicidal risk, auditory impairment, or with a history of neurological disorder, substance abuse, mental retardation, or brain trauma, as well as pregnant women, were excluded. The Korean version of the Beck Depression Inventory (BDI), ${ }^{22}$ the Young Mania Rating Scale (YMRS), ${ }^{23}$ and the Hamilton Depression Rating Scale (HDRS)-21 $1^{24}$ were used. Subjects were classified as euthymic if they had a BDI score of 10 points or under, an HDRS score of 13 points or under, and a YMRS score of 12 points or fewer, for at least 2 months before the LDAEP was measured. Subjects were classified as mania if they had a YMRS of 14 points or more before the LDAEP was measured. Subjects were classified as depression if they had a BDI of 17 points or more, and an HDRS score of 18 points or more, for at least 1 week before the LDAEP was measured. Inter-rater reliabilities were maintained high (kappa $>0.8$ ) in the measurements using the YMRS and HDRS by using a regular symptom rating training program. Written informed consent was obtained from all of the subjects, and the study protocol was approved by the ethics committee of the Inje University Ilsanpaik Hospital.

Patients with bipolar disorder were taking mood-stabilizing agents (valproic acid, 100-1,500 mg, n=21; lithium, 300$900 \mathrm{mg}, \mathrm{n}=12$; carbamazepine, $300 \mathrm{mg}, \mathrm{n}=1$ ) with or without atypical antipsychotics (quetiapine, $n=21$; risperidone, $n=5$; olanzapine, $n=3$; aripiprazole, $n=3$; amisulpride, $n=1$; blonanserin, $n=1$ ). Patients with schizophrenia were taking 1 of the atypical antipsychotics (risperidone, $n=14$; quetiapine, $n=10$; paliperidone, $n=5$; olanzapine, $n=4$; amisulpride, $n=4$; aripiprazole, $n=2$; blonanserin, $n=2$; clozapine, $n=2$ ). When converted to an equivalent dose of chlorpromazine, the average dose of antipsychotics in schizophrenia patients was $513 \mathrm{mg}$.

Among the bipolar subgroups, the number of patients who were taking antipsychotics in bipolar depression was 5; in mania, 15; and in euthymia, 10. The number of patients taking antidepressants in bipolar depression was 6; in mania, 0 ; and in euthymia, 1 . Mood stabilizers were taken by 6 patients in bipolar depression; in mania, 15; and in euthymia, 10. When converted to an equivalent dose of chlorpromazine, the average 
dose of antipsychotics in bipolar depression was $293 \mathrm{mg}$; in mania, $497 \mathrm{mg}$; and in euthymia, $253 \mathrm{mg}$.

\section{Electrophysiological assessment and data preparation}

All of the subjects were seated in a comfortable chair in a sound-attenuated room. Auditory stimulation comprised 1,000 stimuli with an interstimulus interval that was randomized between 500 and $900 \mathrm{~ms}$. Tones of $1,000 \mathrm{~Hz}$ and 80 -ms duration (10-ms rise and 10-ms fall) were presented at 5 intensities: $55,65,75,85$, and $95 \mathrm{~dB}$ SPL through MDR-D777 headphones (Sony, Tokyo, Japan). These stimuli were generated by EPrime software (Psychology Software Tools, Pittsburgh, USA). Electroencephalogram (EEG) data were recorded and amplified using a Neuroscan SynAmp amplifier (Compumedics USA, El Paso, TX, USA) with $64 \mathrm{Ag}-\mathrm{AgCl}$ electrodes (FP1, FPZ, FP2, AF3, AF4, F7, F5, F3, F1, FZ, F2, F4, F6, F8, FT7, FC5, FC3, FC1, FCZ, FC2, FC4, FC6, FT8, T7, C5, C3, C1, CZ, C2, C4, C6, T8, TP7, CP5, CP3, CP1, CPZ, CP2, CP4, CP6, TP8, P7, P5, P3, P1, PZ, P2, P4, P6, P8, PO7, PO5, PO3, $\mathrm{POZ}, \mathrm{PO} 4, \mathrm{PO} 6, \mathrm{PO}$, CB1, O1, OZ, O2, CB2, M1, and M2) mounted in a Quick Cap using a modified 10-20 placement scheme (impedance $<10 \mathrm{k} \Omega$ ). The vertical electro-oculogram (EOG) was recorded using 2 electrodes, one located above, and one below, the right eye. The horizontal EOG was recorded at the outer canthi of each eye. The ground electrode was placed on the forehead, and the reference was located at electrode Cz. EEG data were recorded with a $0.1-100-\mathrm{Hz}$ bandpass filter and a 1,000-Hz sampling rate. EEG data were initially processed using Scan 4.3, and re-referenced offline to an average reference. Eye blinks were removed from the data using standard blink correction algorithms 25 . Trials were rejected if they included significant physiological artifacts (e.g., amplitude exceeding $\pm 70 \mathrm{~V}$ ) at all 62 electrode sites, except for M1 and M2. After artifact removal, baseline correction was conducted by subtracting the mean of $100 \mathrm{~ms}$ of prestimulus data from the post-stimulus data for each trial.

\section{Cortical LDAEP analysis}

Data were band-pass filtered at $0.1-30 \mathrm{~Hz}$ and then epoched to $100 \mathrm{~ms}$ prestimulus and $600 \mathrm{~ms}$ post-stimulus. For 5 sound intensities, and for each subject, the N1 peak (most negative amplitude between 80 and $130 \mathrm{~ms}$ from the stimulus) and P2 peak (most positive peak between 130 and $230 \mathrm{~ms}$ from the stimulus) were then determined at the $\mathrm{Cz}$ electrode. The $\mathrm{Cz}$ electrode was chosen on the basis of a previous study 26 that reported the LDAEP values from $\mathrm{Fz}, \mathrm{Cz}$, and $\mathrm{Pz}$ in different stages of schizophrenia, as well as healthy controls. In their results, the $\mathrm{Cz}$ electrode produced the most prominent LDAEP value, compared with the $\mathrm{Fz}$ and $\mathrm{Pz}$ electrodes, in every subject. The peak-to-peak N1/P2 amplitudes were calculated for the 5 stimulus intensities, and the LDAEP was calculated as the slope of the linear regression.

\section{Source LDAEP analysis}

On the basis of the averaged, scalp-recorded electric potential, standardized low-resolution brain electromagnetic tomography (sLORETA) was used to estimate current density. ${ }^{27}$ The sLORETA technique estimates the standardized source current density by using the realistic 3-shell head model, on the basis of the Montreal Neurological Institute (MNI) 152 template provided by the Brain Imaging Center of the MNI, under the assumption that the activity at any single neuron should be highly synchronized to the activity of its closest neighbors. ${ }^{28}$ The solution space is restricted to the cortical gray matter and hippocampus of the head model and partitioned into 6239 voxels at a spatial resolution of $5 \mathrm{~mm}$. Anatomical labels, such as Brodmann areas (BAs), are provided by the use of an appropriate transformation from MNI to Talairach space. ${ }^{29}$ The loudness dependence of the source activity (source LDAEP) was determined by calculating current source densities for each subject and each sound pressure level. Two electrodes (M1, M2) were not used in the sLORETA analysis because these electrode locations are not supported by the sLORETA software. The calculated standardized current density was averaged between 60 and $240 \mathrm{~ms}$ post-stimulus from the primary auditory cortex (BA41), in accordance with a previous study. ${ }^{2,30}$ We calculated the 3 values of current density for the left, right, and averaged data- 1 from both hemispheres over the voxels that fall under the primary auditory cortex. The source LDAEP was calculated as the slope of the linear regression of current density of BA41 for the 5 stimulus intensities.

\section{Statistical analysis}

Diagnosis was classified in 3 different ways: 3 groups (bipolar disorder, schizophrenia, healthy controls), 5 groups (bipolar mania, bipolar depression, bipolar euthymia, schizophrenia, and healthy controls), and as 3 bipolar subgroups (bipolar mania, bipolar depression, bipolar euthymia). Group differences were assessed by one-way ANOVA with diagnostic groups as independent factors and LDAEP as the dependent variable. There is compelling evidence to indicate gender differences in serotonergic function among depressed patients. ${ }^{31-33}$ Gender could therefore be a serious influencing factor on LDAEP. If the gender distribution showed a significant difference among groups, univariate ANOVA would be performed with diagnostic groups as independent factors, LDAEP as dependent variable, and gender as a covariate. Post hoc comparison was performed using the least significant difference method. Uncorrected values of means and standard deviations were presented. 
Furthermore, bipolar disorder patients were divided into those who presented psychotic features during the current mood episode and those who did not, or those having a history of psychotic features and those who do not. An independent ttest was performed for 2 bipolar patient groups. For correlation analysis between symptom severity and bipolar subgroups, Spearman analysis was conducted.

\section{RESULTS}

\section{Demographic characteristics}

Table 1 presents the distribution of demographic characteristics and the mean score of the symptoms scales for each mental disorder. There was a significant gender difference among the 3 groups ( $\mathrm{p}=0.033)$, but not among 5 groups $(\mathrm{p}=0.070)$ or the 3 bipolar subgroups $(\mathrm{p}=0.366)$.

\section{Cortical LDAEP}

In 3 group analysis, after controlling for gender effects, there were no significant differences among groups $(\mathrm{F}=12.027, \mathrm{df}=$ $2, \mathrm{p}=0.077)$. LDAEP exhibited a tendency of decreasing strength in the order of healthy controls, bipolar disorder patients, and schizophrenia patients.

In 5 group analysis, there was a significant difference in LDAEP among groups $(\mathrm{F}=6.217, \mathrm{df}=4, \mathrm{p}=0.000$; Figure 1). Analysis of LDAEP revealed a tendency of decreasing strength in the patients in the following order: healthy controls, patients with bipolar euthymia, patients with bipolar depression, patients with bipolar mania and those with schizophrenia. A post hoc analysis revealed that LDAEP was significantly stronger in patients with bipolar depression than in those with schizophrenia $(0.86 \pm 0.69$ vs. $0.46 \pm 0.38 \mathrm{uV} / 10 \mathrm{~dB}, \mathrm{p}=0.027)$, stronger in bipolar euthymia than in schizophrenia ( $1.06 \pm 0.54$ vs. $0.46 \pm 0.38 \mathrm{uV} / 10 \mathrm{~dB}, \mathrm{p}=0.001)$, stronger in healthy controls relative to patients with schizophrenia $(1.07 \pm 0.51$ vs. $0.46 \pm 0.38$ $\mathrm{uV} / 10 \mathrm{~dB}, \mathrm{p}=0.000$ ), and stronger in healthy controls relative to patients with bipolar mania $(1.07 \pm 0.51$ vs. $0.74 \pm 0.47 \mathrm{uV} / 10$ $\mathrm{dB}, \mathrm{p}=0.046$ ).

In bipolar subgroups analysis, patients with bipolar depression, bipolar mania, and bipolar euthymia were compared; there were no significant differences $(\mathrm{F}=0.999$, $\mathrm{df}=2, \mathrm{p}=0.379)$.

Among patients with bipolar disorder, patients were divided into those presenting psychotic features during the current mood episode and those who did not, as well as into those with a history of psychotic features and those without. A t-test for independent samples indicated that the strength of the LDAEP did not differ significantly between the 2 groups in either case $(t=0.326, p=0.746$; and $t=0.98, p=0.334)$. Among the subgroups of bipolar disorder, the BDI and HDRS scores of bipolar depression and euthymia did not significantly correlate with LDAEP. Similarly, the YMRS score of bipolar mania did not significantly correlate with LDAEP.

Table 1. Demographic data of the subjects

\begin{tabular}{|c|c|c|c|c|c|c|}
\hline & \multicolumn{3}{|c|}{$\mathrm{BD}$} & \multirow{2}{*}{ SPR } & \multirow{2}{*}{$\mathrm{HC}$} & \multirow{2}{*}{$\mathrm{p}$ value } \\
\hline & Depression & Mania & Euthymia & & & \\
\hline Number of subjects (N) & 10 & 15 & 10 & 32 & 22 & \\
\hline Gender (male/female) & $2 / 8$ & $7 / 8$ & $3 / 7$ & $19 / 13$ & $6 / 16$ & $0.033,{ }^{*} 0.070^{\dagger}, 0366^{\ddagger}$ \\
\hline Age (years) & $33.1 \pm 9.3$ & $35.9 \pm 9.6$ & $38.9 \pm 11.4$ & $33.6 \pm 9.3$ & $30.1 \pm 12.4$ & $0.221^{\S}$ \\
\hline Duration of illness (years) & $5.6 \pm 5.4$ & $7.8 \pm 6.7$ & $6.9 \pm 4.1$ & $6.4 \pm 6.2$ & & $0.803^{\S}$ \\
\hline Total episodes $(\mathrm{N})$ & $1.5 \pm 1.3$ & $3.9 \pm 3.2$ & $2.7 \pm 2.1$ & & & $0.223 \S$ \\
\hline Depressed episodes $(\mathrm{N})$ & $1.2 \pm 0.9$ & $0.73 \pm 1.2$ & $1 \pm 0.9$ & & & $0.541^{\S}$ \\
\hline Manic episodes (N) & $0.3 \pm 0.5$ & $3 \pm 2.8$ & $1.8 \pm 1.7$ & & & $0.058 \S$ \\
\hline Number of subjects using antipsychotics (\%) & $5(50)$ & $15(100)$ & $10(100)$ & $32(100)$ & & \\
\hline CPZ-equivalent dose for 1 patient (mg) & 293 & 497 & 253 & 513 & & \\
\hline Number of subjects using antidepressants (\%) & $6(60)$ & $0(0)$ & $1(10)$ & $1(3)$ & & \\
\hline Number of subjects using mood stabilizer (\%) & $6(60)$ & $15(100)$ & $10(100)$ & $6(19)$ & & \\
\hline HDRS score & $23.2 \pm 5.7$ & & $8.6 \pm 3.1$ & & & $0.000^{\pi}$ \\
\hline BDI score & $22 \pm 5.2$ & & $6.3 \pm 2.3$ & & & $0.000^{\pi}$ \\
\hline YMRS score & & $24.2 \pm 6.1$ & & & & \\
\hline PANSS score & & & & $98 \pm 21.8$ & & \\
\hline
\end{tabular}




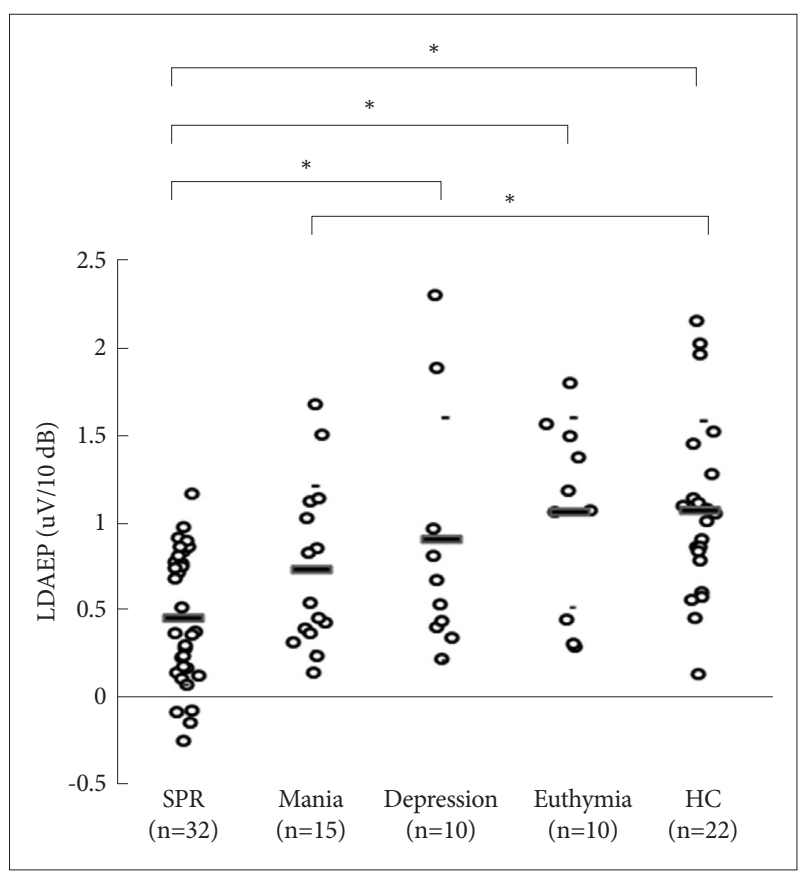

Figure 1. Comparison of loudness dependence of the auditory evoked potential (LDAEP) among schizophrenia (SPR), bipolar mania (Mania), bipolar depression (Depression), bipolar euthymia (Euthymia), and healthy controls ( $\mathrm{HC}$ ). Mean values were presented as horizontal bars. There was a strong trend of significant effect of the diagnostic groups. *represents a statistically significant difference between 2 groups $(p<0.05)$.

\section{Source LDAEP}

In 3 group analysis, after controlling for gender effects, there were no significant group differences in the left hemisphere $(\mathrm{F}=1.534, \mathrm{df}=2, \mathrm{p}=0.395)$, right hemisphere $(\mathrm{F}=3.661, \mathrm{df}=2$, $\mathrm{p}=0.215)$, or average $(\mathrm{F}=2.518, \mathrm{df}=2, \mathrm{p}=0.284)$.

In 5 group analysis, there was a significant group difference in the averaged source LDAEP in both hemispheres $(\mathrm{F}=3.448$, $\mathrm{df}=4, \mathrm{p}=0.012$; Figure 2). A post hoc analysis revealed that the averaged source LDAEP was significantly stronger in patients with bipolar depression than in those with schizophrenia $(0.047 \pm 0.060$ vs. $0.009 \pm 0.057$ current value $/ 10 \mathrm{~dB}, \mathrm{p}=$ 0.050 ), stronger in bipolar euthymia than in schizophrenia $(0.073 \pm 0.054$ vs. $0.009 \pm 0.057$ current value $/ 10 \mathrm{~dB}, \mathrm{p}=0.001)$, stronger in healthy controls than in patients with schizophrenia $(0.040 \pm 0.049$ vs. $0.009 \pm 0.057$ current value $/ 10 \mathrm{~dB}, \mathrm{p}=$ 0.035 ), and stronger in bipolar euthymia than in bipolar mania $(0.073 \pm 0.054$ vs. $0.024 \pm 0.032$ current value $/ 10 \mathrm{~dB}, \mathrm{p}=$ $0.025)$. However, there was no significant primary effect of group differences in the left hemisphere $(\mathrm{F}=2.179, \mathrm{df}=4$, $\mathrm{p}=0.078)$ or right hemisphere $(\mathrm{F}=2.359, \mathrm{df}=4, \mathrm{p}=0.060)$.

In the analysis of the 3 bipolar subgroups, there were no significant group differences in left hemisphere $(\mathrm{F} 1.578, \mathrm{df}=$ $2, \mathrm{p}=0.222)$, right hemisphere $(\mathrm{F}=2.676, \mathrm{df}=2, \mathrm{p}=0.084)$, or averaged $(\mathrm{F}=3.028, \mathrm{df}=2, \mathrm{p}=0.062)$.

There was a significant difference in the averaged source

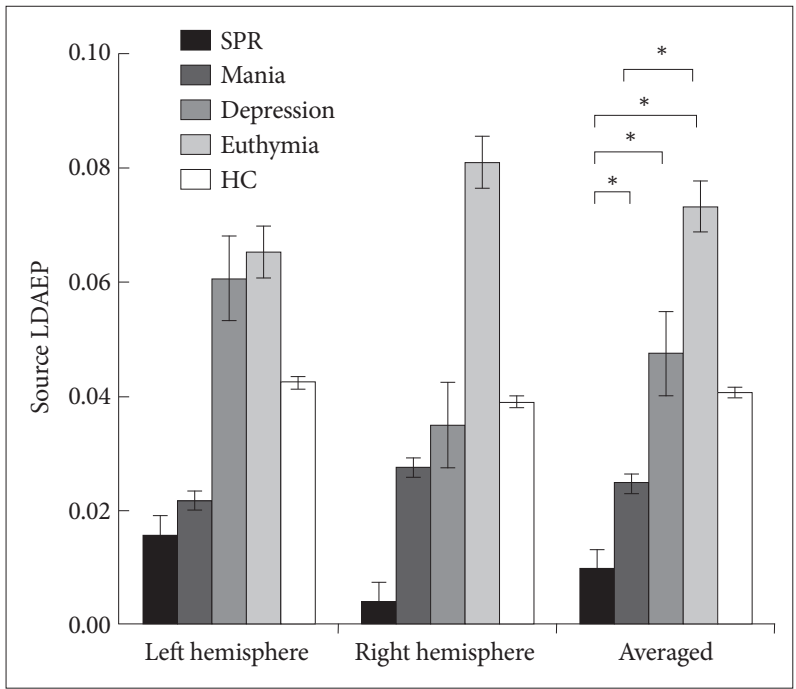

Figure 2. Comparison of source loudness dependence of the auditory evoked potential (LDAEP) among schizophrenia (SPR), bipolar mania (Mania), bipolar depression (Depression), bipolar euthymia (Euthymia), and healthy controls (HC). The LDAEP of the current source density of the primary auditory cortex (BA41) was calculated individually for the left and right hemispheres, and averaged across both hemispheres. Error bars are presented. There were no significant primary effects of the diagnostic groups. *represents a statistically significant difference between 2 groups $(p<0.05)$.

LDAEP of both hemispheres between bipolar disorder with psychotic features and bipolar disorder without psychotic features in the current mood episode ( $0.017 \pm 0.034$ vs. $0.056 \pm$ $0.053, \mathrm{p}=0.038$; Figure 3 ). However, when comparing a history of psychotic features, there was no significant difference.

In the bipolar disorder depression and euthymia groups, the BDI score showed significant correlations with the averaged source LDAEP ( $r h o=-0.445, \mathrm{p}=0.049$; Figure 4 ). The HDRS score also showed a trend of significant correlation with the averaged source LDAEP ( $r$ o $=-0.437$, $\mathrm{p}=0.054$; Figure 4). However, the YMRS score of bipolar mania did not significantly correlate with source LDAEPs.

\section{DISCUSSION}

Our results revealed that the strength of LDAEP varied according to current mood status, and was strongest in descending order of euthymia, depression, and mania. Patients with bipolar disorder who exhibited psychotic features in their current mood episode demonstrated weaker source LDAEP compared with that of bipolar disorder without psychotic features. Furthermore, the BDI and HDRS scores were negatively correlated with source LDAEP strength in bipolar euthymia and depression.

The LDAEP of patients with bipolar disorder varied according to current mood status. Bipolar mania showed the weakest LDAEP-values as low as those of schizophrenia patients. Me- 
anwhile, bipolar euthymia exhibited comparable LDAEP strength to healthy controls. Previous results from our laboratory revealed that the LDAEP in bipolar disorder is as low as that of schizophrenia patients. ${ }^{34}$ However, the present results revealed that this is not true in all cases. This discrepancy may be explained by the different distribution of bipolar disorder subgroups between the 2 studies. In the study by Park et al. ${ }^{34}$ the number of patients with bipolar mania was 28 (75.6\%), while patients with bipolar depression was just 9 (24.4\%). In

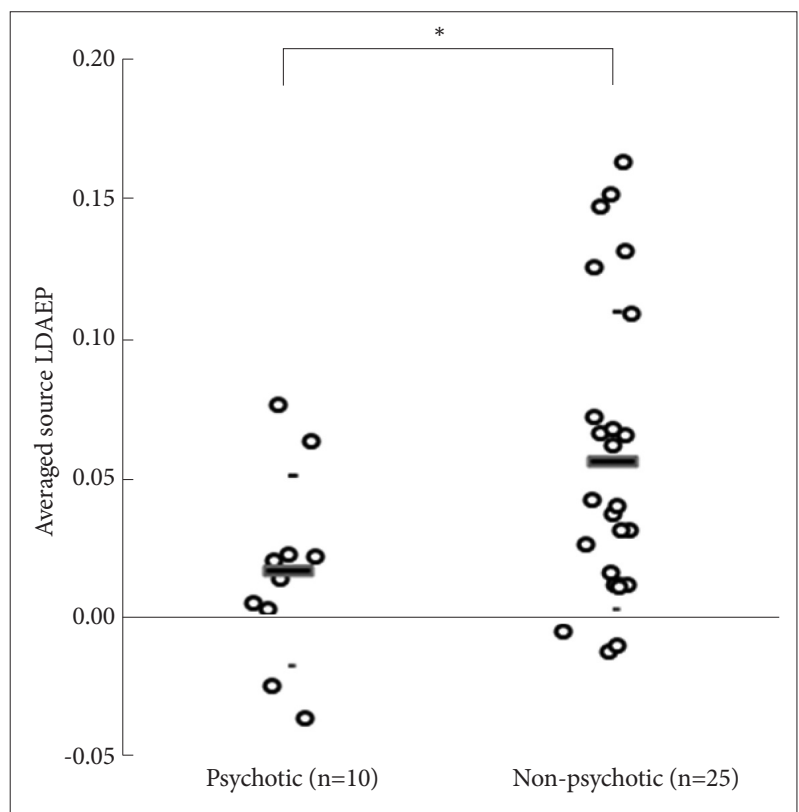

Figure 3. Comparison of averaged source loudness dependence of the auditory evoked potential (LDAEP) of both hemispheres between bipolar disorder with psychotic features and without psychotic features in the current episode. Mean values were presented as horizontal bars. *represents a statistically significant difference between 2 groups $(p<0.05)$. the current study, the proportions of bipolar mood statuses were as follows: mania, 15 (42.8\%); depression, 10 (28.5\%); and euthymia, 10 (28.5\%). Bipolar depression and euthymia exhibited stronger LDAEP relative to bipolar mania. A higher proportion of bipolar depression and euthymia in the current study, compared with the cohort in Park et al. ${ }^{34}$ would contribute to strengthen the overall LDAEP in the bipolar group. The current results suggest that different bipolar subgroups have different LDAEP properties, and reflect different pathophysiological backgrounds among bipolar subgroups.

Our results revealed that bipolar mania and bipolar disorder with psychotic features produced weaker LDAEP compared with bipolar euthymia/depression and bipolar disorder without psychotic features. It can be assumed that weaker LDAEP may reflect symptom severity or psychotic tendencies in bipolar disorder. The weak LDAEP observed in schizophrenia patients has been reported in several studies. Park et al. ${ }^{34}$ reported that LDAEP was significantly lower in schizophrenia patients compared with healthy controls. Gudlowski et al. ${ }^{26}$ reported a significantly weaker LDAEP found in prodromal, first-episode, and chronic patients with schizophrenia compared with healthy controls. Juckel et al. ${ }^{35}$ reported that patients with schizophrenia exhibited significantly weaker LDAEP than healthy controls. These results support the hypothesis that psychotic symptoms may correlate with reduced LDAEP. With respect to neurocognitive function, some studies report no difference between bipolar mania and the schizophrenia group. ${ }^{36-38}$ Bipolar manic patients are found to perform as poorly as schizophrenia patients on the perceptual span. ${ }^{36}$ However, there are a number of studies that show that the cognitive function of euthymic patients is largely intact. ${ }^{39-43}$ Some fMRI studies have directly compared activation pat-
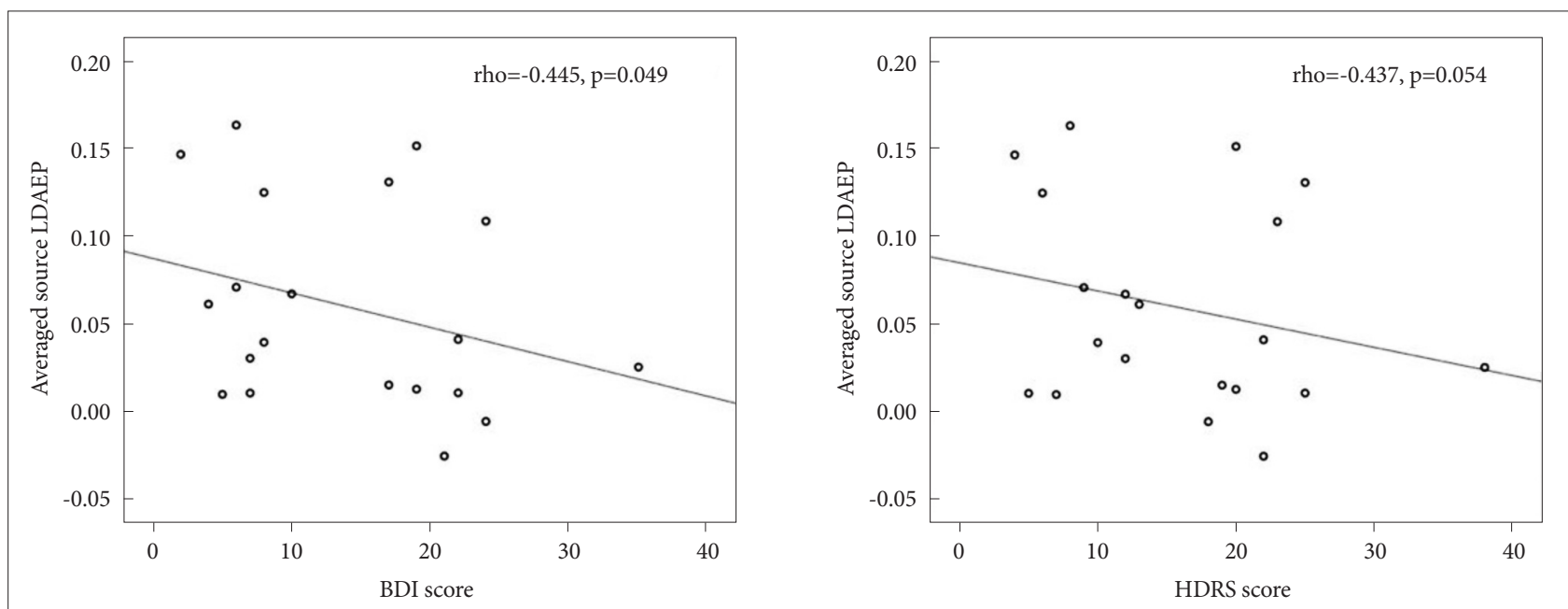

Figure 4. Spearman's correlation between averaged source loudness dependence of the auditory evoked potential (LDAEP) and depressive symptom severity scores in bipolar patients with depressive symptoms or euthymic symptoms $(n=20)$. The Beck Depression Inventory (BDI) score showed significant correlation with averaged source LDAEP $(r=-0.445, p=0.049)$. The Hamilton Depression Rating Scale (HDRS) score also showed a significant correlation with averaged source LDAEP $(r=-0.437, p=0.054)$. 
terns across mood states. ${ }^{44-46}$ Hulvershorn et al. ${ }^{44}$ identified different activation patterns in different phases of bipolar disorder using a facial emotion matching task. The bipolar mania group exhibited decreased left lateral orbitofrontal cortex activation compared with both bipolar euthymia and healthy controls. ${ }^{44}$ Generally, manic patients demonstrated a greater deficit in activating brain regions to emotional and cognitive stimuli compared with depressive and euthymic patients. ${ }^{45,46}$ These results highlight that manic patients have deficits in neuropsychological function and emotion processing compared with euthymic patients, and these distinctive cognitive deficits may reflect the weak LDAEP in bipolar mania.

Our source LDAEP results are consistent with previous evidence regarding serotonergic function in bipolar disorder. Asberg et al. ${ }^{16}$ reported that the absolute levels of 5-HIAA are reduced in the CSF during bipolar depression $(89.5 \pm 38.6 \mathrm{nmol} /$ L) compared with controls $(104.1 \pm 38.3 \mathrm{nmol} / \mathrm{L}) .{ }^{16}$ In the diagnostic subcategory, bipolar I disorder patients tended to have lower 5-HIAA concentrations than unipolar patients and bipolar II disorder patients. ${ }^{16}$ Swann et al. ${ }^{17}$ demonstrated higher 5-HIAA levels in bipolar mania (9 male and 5 female subjects) compared with healthy control subjects (30 male and $32 \mathrm{fe}$ male subjects). The manic female subjects had significantly higher 5-HIAA concentrations $(161.0 \pm 41.2 \mathrm{nmol} / \mathrm{L})$ than $\mathrm{did}$ the control female subjects $(114.0 \pm 37.1 \mathrm{nmol} / \mathrm{L}) .{ }^{17}$ Because CSF 5-HIAA levels may reflect the serotonergic activity of the brain, increased 5-HIAA in bipolar mania would produce weaker LDAEP, and reduced 5-HIAA levels in bipolar depression would produce stronger LDAEP. The averaged source LDAEP values and left hemisphere source LDAEP values are consistent with those reported in previous studies. However, with respect to cortical LDAEP, patients with bipolar depression tended to have lower LDAEP values than the healthy controls. This difference can be explained as follows. First, since the sLORETA technique estimates the standardized source current density, source LDAEP could more appropriately reflect the serotonergic activity of the brain than cortical LDAEP does. ${ }^{27}$ Second, unlike in previous CSF studies on drug-naive bipolar patients, ${ }^{16,17}$ in our study, the effects of medication on LDAEP in the patients could not be excluded. Six out of 10 bipolar depression patients had been chronically treated with antidepressants (bupropion, $n=3$; tianeptine, $n=1$; paroxetine, $n=1$; and escitalopram, $n=1$ ) before LDAEP was estimated. Simmons et al. ${ }^{47}$ reported that chronic administration of SSRI may induce a decrease in LDAEP. Therefore, the LDAEP values may have been underestimated in our study.

From an anatomical perspective, the dorsal raphe nucleus is the origin of most forebrain serotonin innervation. ${ }^{48}$ Matthews and Harrison ${ }^{49}$ used morphometric, immunohistochemical, and molecular methods to compare the dorsal raphe nucleus in postmortem tissue from 50 subjects. The subjects comprised 13 bipolar disorder, 14 major depressive disorder (MDD), and 10 schizophrenia patients, as well as 13 healthy control subjects. In bipolar disorder, the size of serotonergic neurons was decreased in the dorsal raphe nucleus. In MDD, the dorsal raphe nucleus area was decreased, with no difference in cell density or size. However, in schizophrenia, no changes were observed. ${ }^{49}$ Suicide was associated with an increased dorsal raphe nucleus area, and with an increased density and decreased size of serotonergic neurons. ${ }^{49}$ In this study, there was no classification of bipolar patients. However, from this anatomical study, we can assume that schizophrenia patients have relatively preserved serotonergic function (weaker LDAEP) compared with bipolar and MDD patients (stronger LDAEP). These findings are consistent with our current LDAEP results.

Interestingly, our results showed that BDI and HDRS scores were negatively correlated with source LDAEP strength in bipolar euthymia and depression. These findings imply that as depressive symptoms worsen in bipolar disorder patients, the strength of source weakens. The significant correlation between LDAEP and symptom severity was found in sourcelevel LDAEP, but not cortical LDAEP. Furthermore, the cortical LDAEP of healthy controls was the strongest among the 5 groups; however, the source LDAEPs of healthy controls were ranked among the top third. These findings support the conclusion that cortical LDAEP may differ from source LDAEP. Further studies are needed to address this issue.

This study has several limitations. First, we did not control for the use of drugs. Studies on the influence of psychotrophic drugs on LDAEP values report inconsistent results. O’Neill et al. ${ }^{50}$ reported that when healthy participants were tested under 3 acute treatment conditions (placebo, bromocriptine, and pergolide), acute stimulation of the dopamine receptor did not modulate LDAEP. Hitz et al. ${ }^{51}$ reported that acute administration of levodopa did not lead to any significant LDAEP alterations from the baseline measurement values in healthy subjects. Guille et al. ${ }^{52}$ reported that when healthy subjects were tested under 4 acute treatment conditions (placebo, escitalopram, citalopram, and sertraline), the LDAEP values were not altered in any way. Oliva et $\mathrm{al} .{ }^{53}$ reported that when healthy subjects were tested after acute administration of citalopram, reboxetine, and placebo, neither citalopram nor reboxetine modulated the LDAEP values. ${ }^{53}$ These findings suggest that LDAEP is not sensitive to acute changes in dopaminergic or serotonergic neurotransmission. ${ }^{50-53}$ In contrast, Juckel et al. ${ }^{35}$ reported that after chronic administration (4 weeks) of clozapine or olanzapine, LDAEP values tended to increase from the baseline measurements in patients with schizophrenia. Simmons et al. ${ }^{47}$ reported that chronic administration (about 24 
days) of sertraline led to significant reduction in the LDAEP values from the baseline measurements. LDAEP may be more sensitive to chronic changes than to acute or short-term changes in serotonergic neurotransmission. ${ }^{47}$ However, we cannot exclude the effects of medication in this study because the subjects were taking medication chronically to control their symptoms. Second, in the longitudinal process of the illness, bipolar disorder patients experience various episodes alternately, such as mania, depression, or euthymia. Thus, LDAEP changes throughout a long-term period in the same patient cohort would be an interesting avenue to pursue in the future.

\section{Conclusion}

In conclusion, the present study revealed that serotonin function, reflected by LDAEP, varied with current mood status; it was strongest in descending order of euthymia, depression, and mania. Concurrent psychotic features also weaken the LDAEP in bipolar disorder. Furthermore, the severity of the depressive symptom was negatively correlated with source LDAEP strength in bipolar euthymia and depression.

\section{Acknowledgments}

This study was supported by a grant from the Korean Health Technology R\&D Project, Ministry for Health, Welfare, and Family Affairs, Republic of Korea (A070001).

\section{REFERENCES}

1. Hegerl U, Juckel G. Intensity dependence of auditory evoked potentials as an indicator of central serotonergic neurotransmission: a new hypothesis. Biol Psychiatry 1993;33:173-187.

2. Park YM, Kim DW, Kim S, Im CH, Lee SH. The loudness dependence of the auditory evoked potential (LDAEP) as a predictor of the response to escitalopram in patients with generalized anxiety disorder. Psychopharmacology (Berl) 2011;213:625-632.

3. Lee SH, Park GH. Psychophysiological Markers of Anxiety Disorders and Anxiety Symptoms. In: Kalinin V, Editor. Anxiety Disorders. Rijeka: InTech, 2011, p.203-226.

4. Salvadore G, Quiroz JA, Machado-Vieira R, Henter ID, Manji HK, Zarate CA Jr. The neurobiology of the switch process in bipolar disorder: a review. J Clin Psychiatry 2010;71:1488-1501.

5. Lee SH, Kim JH, Lee JH, Kim S, Park YM, Bae SM, et al. Aberrant response of selective serotonin reuptake inhibitor in two patients with high N100 amplitude slope. Korean J Psychopharmacol 2008;19:341347.

6. Park YM, Lee SH, Park EJ. Usefulness of LDAEP to predict tolerability to SSRIs in major depressive disorder: a case report. Psychiatry Investig 2012;9:80-82.

7. Hegerl U, Ulrich G, Muller-Oerlinghausen B. Auditory evoked potentials and response to lithium prophylaxis. Pharmacopsychiatry 1987;20: 213-216.

8. Hegerl U, Wulff H, Muller-Oerlinghausen B. Intensity dependence of auditory evoked potentials and clinical response to prophylactic lithium medication: a replication study. Psychiatry Res 1992;44:181-190.

9. Rohayem J, Bayle JF, Richa S. Predictors of prophylactic response to lithium. Encephale 2008;34:394-399.

10. Hegerl U, Gallinat J, Juckel G. Event-related potentials. Do they reflect central serotonergic neurotransmission and do they predict clinical response to serotonin agonists? J Affect Disord 2001;62:93-100.

11. Mundo E, Walker M, Cate T, Macciardi F, Kennedy JL. The role of serotonin transporter protein gene in antidepressant-induced mania in bipolar disorder: preliminary findings. Arch Gen Psychiatry 2001;58: 539-544.

12. Rao JS, Kellom M, Reese EA, Rapoport SI, Kim HW. Dysregulated glutamate and dopamine transporters in postmortem frontal cortex from bipolar and schizophrenic patients. J Affect Disord 2012;136:63-71.

13. Dean B, Pavey G, McLeod M, Opeskin K, Keks N, Copolov D. A change in the density of $[(3) \mathrm{H}]$ flumazenil, but not $[(3) \mathrm{H}]$ muscimol binding, in Brodmann's Area 9 from subjects with bipolar disorder. J Affect Disord 2001;66:147-158.

14. Sun Y, Zhang L, Johnston NL, Torrey EF, Yolken RH. Serial analysis of gene expression in the frontal cortex of patients with bipolar disorder. Br J Psychiatry Suppl 2001;41:s137-s141.

15. Mahmood T, Silverstone T. Serotonin and bipolar disorder. J Affect Disord 2001;66:1-11.

16. Asberg M, Bertilsson L, Martensson B, Scalia-Tomba GP, Thoren P, Traskman-Bendz L. CSF monoamine metabolites in melancholia. Acta Psychiatr Scand 1984;69:201-219.

17. Swann AC, Secunda S, Davis JM, Robins E, Hanin I, Koslow SH, et al. CSF monoamine metabolites in mania. Am J Psychiatry 1983;140:396400.

18. Thakore JH, O'Keane V, Dinan TG. d-fenfluramine-induced prolactin responses in mania: evidence for serotonergic subsensitivity. Am J Psychiatry 1996;153:1460-1463.

19. Nurnberger JI Jr, Berrettini W, Simmons-Alling S, Lawrence D, Brittain H. Blunted ACTH and cortisol response to afternoon tryptophan infusion in euthymic bipolar patients. Psychiatry Res 1990;31:57-67.

20. First MB, Spitzer RL, Gibbon M, Williams JBW. User's Guide for the Structured Clinical Interview for DSM-IV Axis I Disorders: Clinician Version (SCID-CV). Washington, DC:American Psychiatric Press; 1997.

21. First MB, Gibbon M, Spitzer RL, Williams JBW. User's Guide for the Structured Clinical Interview for DSM-IV Axis II Personality Disorders (SCID-II). Washington, DC: American Psychiatric Press; 1997.

22. Beck AT, Ward CH, Mendelson M, Mock J, Erbaugh J. An inventory for measuring depression. Arch Gen Psychiatry 1961;4:561-571.

23. Young RC, Biggs JT, Ziegler VE, Meyer DA. A rating scale for mania: reliability, validity and sensitivity. Br J Psychiatry 1978;133:429-435.

24. Hamilton M. A rating scale for depression. J Neurol Neurosurg Psychiatry 1960;23:56-62.

25. Semlitsch HV, Anderer P, Schuster P, Presslich O. A solution for reliable and valid reduction of ocular artifacts, applied to the P300 ERP. Psychophysiology 1986;23:695-703.

26. Gudlowski Y, Ozgurdal S, Witthaus H, Gallinat J, Hauser M, Winter C, et al. Serotonergic dysfunction in the prodromal, first-episode and chronic course of schizophrenia as assessed by the loudness dependence of auditory evoked activity. Schizophr Res 2009;109:141-147.

27. Pascual-Marqui RD. Standardized low-resolution brain electromagnetic tomography (sLORETA): technical details. Methods Find Exp Clin Pharmacol 2002;24(Suppl D):5-12.

28. Fuchs M, Kastner J, Wagner M, Hawes S, Ebersole JS. A standardized boundary element method volume conductor model. Clin Neurophysiol 2002;113:702-712.

29. Brett M, Johnsrude IS, Owen AM. The problem of functional localization in the human brain. Nat Rev Neurosci 2002;3:243-249.

30. Mulert C, Juckel G, Augustin H, Hegerl U. Comparison between the analysis of the loudness dependency of the auditory N1/P2 component with LORETA and dipole source analysis in the prediction of treatment response to the selective serotonin reuptake inhibitor citalopram in major depression. Clin Neurophysiol 2002;113:1566-1572.

31. Cosgrove KP, Mazure CM, Staley JK. Evolving knowledge of sex differences in brain structure, function, and chemistry. Biol Psychiatry 
2007;62:847-855.

32. Staley JK, Sanacora G, Tamagnan G, Maciejewski PK, Malison RT, Berman RM, et al. Sex differences in diencephalon serotonin transporter availability in major depression. Biol Psychiatry 2006;59:40-47.

33. Frey BN, Skelin I, Sakai Y, Nishikawa M, Diksic M. Gender differences in alpha-[(11)C]MTrp brain trapping, an index of serotonin synthesis, in medication-free individuals with major depressive disorder: a positron emission tomography study. Psychiatry Res 2010;183:157-166.

34. Park YM, Lee SH, Kim S, Bae SM. The loudness dependence of the auditory evoked potential (LDAEP) in schizophrenia, bipolar disorder, major depressive disorder, anxiety disorder, and healthy controls. Prog Neuropsychopharmacol Biol Psychiatry 2010;34:313-316.

35. Juckel G, Gallinat J, Riedel M, Sokullu S, Schulz C, Moller HJ, et al. Serotonergic dysfunction in schizophrenia assessed by the loudness dependence measure of primary auditory cortex evoked activity. Schizophr Res 2003;64:115-124.

36. Strauss ME, Bohannon WE, Stephens JH, Pauker NE. Perceptual span in schizophrenia and affective disorders. J Nerv Ment Dis 1984;172: 431-435.

37. Strauss ME, Prescott CA, Gutterman DF, Tune LE. Span of apprehension deficits in schizophrenia and mania. Schizophr Bull 1987;13:699704.

38. Nasrallah HA, Tippin J, McCalley-Whitters M. Neurological soft signs in manic patients. A comparison with Schizophrenic and control groups. J Affect Disord 1983;5:45-50.

39. Malhi GS, Ivanovski B, Hadzi-Pavlovic D, Mitchell PB, Vieta E, Sachdev P. Neuropsychological deficits and functional impairment in bipolar depression, hypomania and euthymia. Bipolar Disord 2007;9:114-125.

40. Paradiso S, Lamberty GJ, Garvey MJ, Robinson RG. Cognitive impairment in the euthymic phase of chronic unipolar depression. J Nerv Ment Dis 1997;185:748-754.

41. van Gorp WG, Altshuler L, Theberge DC, Wilkins J, Dixon W. Cognitive impairment in euthymic bipolar patients with and without prior alcohol dependence. A preliminary study. Arch Gen Psychiatry 1998; 55:41-46.

42. Clark L, Kempton MJ, Scarna A, Grasby PM, Goodwin GM. Sustained attention-deficit confirmed in euthymic bipolar disorder but not in firstdegree relatives of bipolar patients or euthymic unipolar depression. Biol Psychiatry 2005;57:183-187.

43. Ferrier IN, Stanton BR, Kelly TP, Scott J. Neuropsychological function in euthymic patients with bipolar disorder. Br J Psychiatry 1999;175: 246-251.

44. Hulvershorn LA, Karne H, Gunn AD, Hartwick SL, Wang Y, Hummer TA, et al. Neural activation during facial emotion processing in unmedicated bipolar depression, euthymia, and mania. Biol Psychiatry 2012;71:603-610.

45. Van der Schot A, Kahn R, Ramsey N, Nolen W, Vink M. Trait and state dependent functional impairments in bipolar disorder. Psychiatry Res 2010;184:135-142.

46. Blumberg HP, Leung HC, Skudlarski P, Lacadie CM, Fredericks CA, Harris BC, et al. A functional magnetic resonance imaging study of bipolar disorder: state- and trait-related dysfunction in ventral prefrontal cortices. Arch Gen Psychiatry 2003;60:601-609.

47. Simmons JG, Nathan PJ, Berger G, Allen NB. Chronic modulation of serotonergic neurotransmission with sertraline attenuates the loudness dependence of the auditory evoked potential in healthy participants. Psychopharmacology (Berl) 2011;217:101-110.

48. Hornung JP. The human raphe nuclei and the serotonergic system. J Chem Neuroanat 2003;26:331-343.

49. Matthews PR, Harrison PJ. A morphometric, immunohistochemical, and in situ hybridization study of the dorsal raphe nucleus in major depression, bipolar disorder, schizophrenia, and suicide. J Affect Disord 2012;137:125-134.

50. O’Neill BV, Croft RJ, Leung S, Guille V, Galloway M, Phan KL, et al. Dopamine receptor stimulation does not modulate the loudness dependence of the auditory evoked potential in humans. Psychopharmacology (Berl) 2006;188:92-99.

51. Hitz K, Heekeren K, Obermann C, Huber T, Juckel G, Kawohl W. Examination of the effect of acute levodopa administration on the loudness dependence of auditory evoked potentials (LDAEP) in humans. Psychopharmacology (Berl) 2012;221:389-396.

52. Guille V, Croft RJ, O’Neill BV, Illic S, Phan KL, Nathan PJ. An examination of acute changes in serotonergic neurotransmission using the loudness dependence measure of auditory cortex evoked activity: effects of citalopram, escitalopram and sertraline. Hum Psychopharmacol 2008;23:231-241.

53. Oliva J, Leung S, Croft RJ, O’Neill BV, O’Kane J, Stout J, et al. The loudness dependence auditory evoked potential is insensitive to acute changes in serotonergic and noradrenergic neurotransmission. Hum Psychopharmacol 2010;25:423-427. 